\title{
CORRECTION
}

View Article Online

View Journal I View Issue

Check for updates

Cite this: J. Mater. Chem. C, 2017. 5, 3855

DOI: $10.1039 / c 7 t c 90056 b$

rsc.li/materials-c

\section{Correction: High quality graphene films with a clean surface prepared by an UV/ozone assisted transfer process}

\author{
Hongyan Sun, ${ }^{\mathrm{ab}}$ Ding Chen, ${ }^{\star a}$ Yuming Wu, ${ }^{\mathrm{b}}$ Qilong Yuan, ${ }^{\mathrm{bc}}$ Liangchao Guo, \\ Dan Dai, ${ }^{b}$ Kuan W. A. Chee, ${ }^{c}$ Yang Xu, ${ }^{d}$ Pei Zhao, ${ }^{e}$ Nan Jiang ${ }^{\star b}$ and Cheng-Te Lin ${ }^{\star b}$
}

Correction for 'High quality graphene films with a clean surface prepared by an UV/Ozone assisted transfer process' by Hongyan Sun et al., J. Mater. Chem. C, 2017, 5, 1880-1884.

The authors regret the omission of one of the authors, Kuan W. A. Chee, from the original manuscript. The corrected list of authors and affiliations for this paper is as shown above.

The Royal Society of Chemistry apologises for these errors and any consequent inconvenience to authors and readers.

\footnotetext{
${ }^{a}$ College of Materials Science and Engineering, Hunan University, Changsha 410082, P. R. China. E-mail: chending@hnu.edu.cn

${ }^{b}$ Key Laboratory of Marine Materials and Related Technologies, Zhejiang Key Laboratory of Marine Materials and Protective Technologies, Ningbo Institute of Materials Technology and Engineering (NIMTE), Chinese Academy of Sciences, Ningbo 315201, China. E-mail: linzhengde@nimte.ac.cn, jiangnan@nimte.ac.cn

${ }^{c}$ Department of Electrical and Electronic Engineering, University of Nottingham, Ningbo 315100, China

${ }^{d}$ Engineering and State Key Laboratory of Silicon Materials, Zhejiang University, Hangzhou, Zhejiang, 310027, China

${ }^{e}$ Institute of Applied Mechanics and Key Laboratory of Soft Machines and Smart Devices of Zhejiang Province, Zhejiang University, Hangzhou 310012, P. R. China
} 